\title{
Letter regarding article by Wei et al.: Comparison of artificial total disc replacement versus fusion for lumbar degenerative disc disease: a meta-analysis of randomised controlled trials
}

\author{
Qiang Wang • Jing-cheng Wang • Chuan-zhi Xiong
}

Received: 16 August 2013 / Accepted: 8 October 2013 / Published online: 1 November 2013

(C) Springer-Verlag Berlin Heidelberg 2013

Dear Editor,

In a recent issue of International Orthopaedics, Wei and his colleagues published an article entitled "Comparison of artificial total disc replacement versus fusion for lumbar degenerative disc disease: a meta-analysis of randomised controlled trials". In this study, the authors performed a meta-analysis of six randomised controlled trials to compare artificial total disc replacement (TDR) with fusion for treatment of lumbar degenerative disc disease (LDDD). The authors concluded that TDR has significant safety and efficacy comparable to lumbar fusion in the treatment of LDDD at two-year follow-up. However, we have several opinions that we would like to communicate to the authors.

First, the publication language was limited to English in the meta-analysis. Therefore, there might exist a potential language bias in their meta-analysis. We suggest that there be no language restriction for the included studies to reduce the bias.

Second, the authors used an inverse variance (IV)-fixed effects model to pool the data in evaluating complication and re-operation rate $\left(I^{2}=63 \%\right.$ or $\left.I^{2}=51 \%\right)$ for TDR and fusion groups at two years, while they write a "randomised effects model" in the results part. We suggest that "DerSimonian and Laird random effects model" should be used to evaluate complication and reoperation rate.

Third, it is not appropriate that summary mean difference (MD) of intra-operative blood loss and operating time were derived using the method of the IV-random effects model (Figs. 4-7). However, studies should be combined by using the DerSimonian and Laird random effects model, which considers both within- and between-study variations [2].

Fourth, there are various types in either disc or fusion for LDDD, which would lead to different outcomes. If possible, we suggest that a meta-analysis of artificial TDR be used with different disc versus fusion and various surgical approaches for LDDD.

Thanks go to the authors for their contribution to supplying us with a comparison of artificial TDR versus fusion for LDDD. However, further studies based on more carefully and scientifically designed RCTs with large samples and long-term follow-up are still needed to assess artificial TDR versus fusion for LDDD.

\section{References}

1. Wei J, Song Y, Sun L, Lv C (2013) Comparison of artificial total disc replacement versus fusion for lumbar degenerative disc disease: a meta-analysis of randomised controlled trials. Int Orthop 37(7): $1315-1325$

2. DerSimonian R, Laird N (1986) Meta-analysis in clinical trials. Control Clin Trials 7:177-188
Q. Wang $\cdot$ J.-c. Wang $\cdot$ C.-z. Xiong $(\bowtie)$

Department of Orthopaedics, Subei People's Hospital of Jiangsu

Province, Clinical Medical College of Yangzhou University,

Yangzhou 225000, Jiangsu Province, People's Republic of China

e-mail: czxiong13@126.com 